\title{
Improvements of Ultra-High Molecular Weight Polyethylene Mechanical Properties by Nitrogen Plasma Immersion Ion Implantation
}

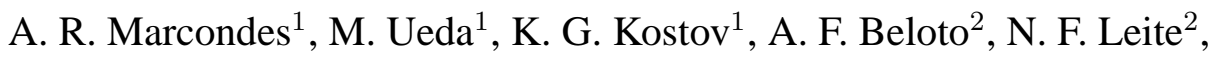 \\ G. F. Gomes ${ }^{1}$, and C. M. Lepienski ${ }^{3}$ \\ ${ }^{1}$ Laboratório Associado de Plasma, Instituto de Pesquisas Espaciais (INPE) \\ São José dos Campos, C.P. 515, ZIP 12201-970, SP, Brazil \\ ${ }^{2}$ Laboratorio Associado de Materiais e Sensores, Instituto de Pesquisas Espaciais (INPE) \\ São José dos Campos, C.P. 515, ZIP 12201-970, SP, Brazil \\ ${ }^{3}$ Universidade Federal do Paraná, Setor de Ciências Exatas, Bloco II, Depto de Física \\ Curitiba, C.P. 19044, ZIP 81531-990, PR, Brazil
}

Received on 29 January, 2004; revised version received on 7 May, 2004

\begin{abstract}
Nitrogen Plasma Immersion Ion Implantation (PIII) has been used to modify the surface chemical structure of Ultra High Molecular Weight Polyethylene (UHMWPE). Grinding and polishing processes based on abrasive papers and alumina pastes have been evaluated with regard to their results on the improvement of polymer surface roughness, which has shown to be of crucial importance for hardness characterization. Raman spectroscopy, XPS, and Nanoindentation tests were used to characterize the modified surfaces. Experimental results has shown that UHMWPE surface mechanical properties such as hardness and elastic modulus can be improved by induced chain cross-linking between the macromolecules on the polymer surface caused by nitrogen PIII. The new material formed on the surface is Diamond Like Carbon (DLC). As a significant improvement in hardness was obtained by DLC synthesis on the treated surface, it is expected a dramatic improvement of abrasion resistance and overall durability of prostheses made with PIII treated UHMWPE.
\end{abstract}

\section{Introduction}

Total joint replacement has become an effective and popular surgical technique for restoring function to patients whose joint have been afflicted by major trauma or joint degenerative diseases. Millions of patients have been fitted with total joint prostheses over the past three decades. A majority of these prostheses have a metal surface articulating against a second component of ultra-high molecular weight polyethylene (UHMWPE). UHMWPE has evolved as the dominant material of choice in total joint prostheses because of its biocompatibility, relative superior friction, wear characteristics, ductility and impact load damping.

The primary problem with UHMWPE in total hip joints is the constant generation of submicron particle debris that overwhelms the body's ability to remove the material. The polyethylene wear particles generated at the articular surface have been recognized as a long-term cause of loosing and failure of the artificial hip joint due to osteolysis (local bone loss) [1]. Therefore it is essential to quantitatively minimize the polyethylene wear particles in hip joints.

Plasma immersion ion implantation (PIII) is an emerging technology that has been used for surface treatment of several kinds of engineering materials, from metals to ceramics. Nowadays it has been used with increasing interest in the field of polymeric materials, specifically those for prostheses, since it offers the possibility of performing three-dimensional ion implantation in geometrically complex parts without the need for workpiece manipulation. PIII offers yet some additional advantages over the conventional ion beam implantation, which has been successfully used to modify the surface of polymers improving their tribological properties [2]. Among these advantages we can mention the relative simplicity of the process, high ions doses, low or moderate ions energy (100 keV or less) and the possibility of large area ion implantation at a reasonable cost.

In the PIII, the workpice to be treated is immersed in plasma from which the implantation species are extracted. Negative high-voltage pulses are applied to the samples. Ions from the plasma are accelerated toward the substrates and are then implanted into the target.

Attempts to use PIII to implant ions in insulating materials such as polymers have presented some technical problems due to surface charge accumulation and voltage reduction across the dielectric. To avoid these problems it has been proposed to conduct PIII treatment over nonconducting material with the use of a metallic grid positioned some millimeters above the sample. So the metallic grid can be connected to the high-voltage pulser instead of the dielectric. Ions accelerated toward the grid, pass through the apertures and are implanted into the insulating material.

Some recent studies $[3,4]$ have demonstrated that PIII is a very promising surface engineering technique to enhance the wear resistance of UHMWPE. 
TABLE 1. Grinding and polishing sequences for the samples surface treatment.

\begin{tabular}{lll}
\hline & Pre-treatment 1 & Pre-treatment 2 \\
\hline Grinding with abrasive papers \#s & $240 / 400 / 600 / 1200$ & $240 / 400 / 600 / 1200$ \\
\hline Polishing on alumina paste \#s & $9 \mu \mathrm{m} / 2 \mu \mathrm{m}$ & $9 \mu \mathrm{m} / 2 \mu \mathrm{m} / 0.3 \mu \mathrm{m} / 0.06 \mu \mathrm{m} / 0.02 \mu \mathrm{m}$ \\
\hline
\end{tabular}

Surface mechanical properties of UHMWPE can be specially improved when Diamond-like carbon (DLC), also known as amorphous hydrogenated carbon $(\mathrm{a}-\mathrm{C}: \mathrm{H})$, is formed in the surface of that polymer. DLC is a class of materials with excellent mechanical, tribological and biological properties. Obviously those unique combination of properties depend on DLC microstructure, which is commonly considered as an amorphous mixture of $\mathrm{sp}^{2}$-type trigonal bonds, and $\mathrm{sp}^{3}$-type tetrahedral bonds hybridized carbon atoms coexisting with $20-40 \%$ of hydrogen. The group of hydrogen-free carbon materials with strong chemical bonding composed by an arrangement of atoms with a high $\mathrm{sp}^{3} / \mathrm{sp}^{2}$ ratio incorporated in an amorphous structure is presently defined as DLC materials [5]. The quality of DLC films is strongly dependent on the $\mathrm{sp}^{3} / \mathrm{sp}^{2}$ carbon atoms ratio. Low $\mathrm{sp}^{3} / \mathrm{sp}^{2}$ ratio approaches the characteristics of DLC film from those of the graphite and high $\mathrm{sp}^{3} / \mathrm{sp}^{2}$ ratio from those of diamond.

The present study was directed towards improving mechanical properties of UHMWPE employing PIII technique. The mechanism involved in the chemical structure change are investigated by Raman and XPS characterization and the surface mechanical properties modifications are evaluated by nanoindentation testing.

\section{Experimental details}

\subsection{Materials}

In this study it was used a medical grade of UHMWPE, GUR 1020, which was supplied by BAUMER (Brazil). The gross sample was in the form of $25 \mathrm{~mm}$ diameter extruded and annealed rod. The density of homogeneous material and average molecular weight, quoted by the manufacturer, are $0.935 \mathrm{~g} / \mathrm{cm}^{3}$ and $3.5 \times 10^{6} \mathrm{~g} / \mathrm{mol}$, respectively. Crystallinity was estimated to be about $50 \%$ using X-ray diffraction method. The rod was cut into discs of approximately 2 $\mathrm{mm}$ in thickness that were grounded in one of their surfaces with $\mathrm{SiC}$ abrasive papers and after that polished on alumina pastes according to two different sequences. The sequences used for grounding and polishing are shown in Table 1. Sequence 2 is more rigorous in terms of the roughness decrease.

\subsection{PIII treatment and characterization}

The samples had their surface treated using the PIII system at the Plasma Associated Laboratory, INPE, Brazil, described elsewhere [6]. The nitrogen plasma was generated by a DC glow discharge with controlled floating plasma potential.

A conductive grid placed some millimeters above the target and connected to a high voltage pulser (RUP4, Germany) was used to permit that nitrogen ions were accelerated enough to be implanted into the polymer surface.

The treatment conditions were the following: working gas pressure at $6.8 \times 10^{-2} \mathrm{~Pa}$, pulses of $10 \mu \mathrm{s}$ in length, 10 $\mathrm{kV}$ in magnitude and frequency of $100 \mathrm{~Hz}$, plasma density of $10^{9} \mathrm{~cm}^{-3}$, plasma temperature of $5.0 \mathrm{eV}$ and treatment time of 30 minutes.

After ion implantation, the surface morphology of the samples was examined using optical microscopy. The structural changes of polymer surface were analyzed through micro-Raman spectroscopy (Renishaw2000) with $514.5 \mathrm{~nm}$ Ar+ laser light and X-ray photoelectron spectroscopy (Kratos XSAM HS). The hardness and elastic modulus of PIIItreated samples were studied employing Tribo Scope Nanomechanical Indentation tester from Hysitron Inc.

\section{Experimental results and discussi- ons}

\subsection{Characterization by Raman Spectroscopy}

It was observed that the surface color of the treated samples had changed from shining ivory-white to brownish yellow. This color modification is probably due to two different processes involving both thermal effect and dehydrogenation [7]. Thermal effect occurs when highly energetic ions pass trough the UHMWPE surface and dissipate some of their energy as heat onto the treated surface. Dehydrogenation occurs when hydrogen atoms are removed from the polymer chains as a result of the ion bombardment. The broken carbon-hydrogen bonds lead to an intensive formation of conjugated carbon-carbon double bonds to which surface color is sensitive.

It is shown in Fig. 1 the extended Raman spectra for both implanted and unimplanted UHMWPE samples. For the unimplanted samples several sharp peaks at 1060, 1127, 1293, 1440, 2722, 2846 and $2882 \mathrm{~cm}^{-1}$ can be observed. Carbon-carbon skeletal stretching is responsible for the weak intensities of the bands at 1060 and $1127 \mathrm{~cm}^{-1}$. The band at $1293 \mathrm{~cm}^{-1}$ with medium intensity is $-\mathrm{CH}_{2}-$ inphase twisting mode and the band at $1440 \mathrm{~cm}^{-1}$ originates from the $-\mathrm{CH}_{2}-$ deformation stretching. The strong intensities in the bands at 2846 and $2882 \mathrm{~cm}^{-1}$ can be attributed to the symmetric and asymmetric $\mathrm{CH}_{2}$ stretchings [8]. Usually, the band at $2722 \mathrm{~cm}^{-1}$ cannot be observed in the Raman spectrum of polyethylene $\left[-\mathrm{CH}_{2}-\right]_{n}$. The band may 
be ascribed to the $-\mathrm{CH}(=\mathrm{O})$ stretching caused by oxygen contamination on the surface.

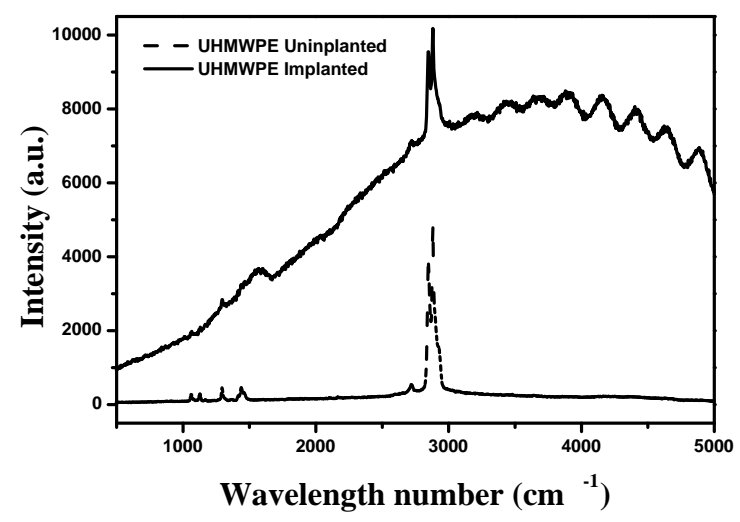

Figure 1. Extended Raman spectra of UHMWPE.

We can see that implantation clearly enhances the effect of photoluminescence on the Raman spectrum, which can be ascribed to an increase of defects in the polymer crystal caused by the implantation of the energetic ions. The reduction of the intensities of bands at 2846 and $2882 \mathrm{~cm}^{-1}$ indicates that the hydrogen content in the surface decreases [2], which is in agreement with our theory about the color change.

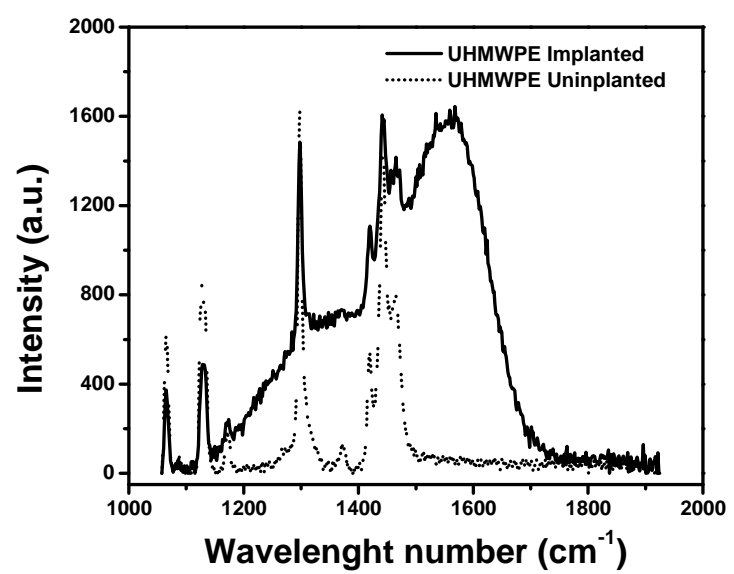

Figure 2. Baseline corrected Raman spectra of implanted and uninplanted UHMWPE samples.

The baseline corrected Raman spectra shown in Fig. 2 gives us some important information about the surface structural modification. In that figure it can be seen that the relative intensity of the bands at 1060, 1127, 1293 and 1440 $\mathrm{cm}^{-1}$ are all decreased as a result of the ions implantation. This suggests that the chemical structure of UHMWPE has changed with the implantation. Especially, the decrease of relative intensity of the peak at $1293 \mathrm{~cm}^{-1}$ indicates that the polymer chain is broken [2] and its length becomes much smaller than the original chain length as the relative intensity of the band at $1293 \mathrm{~cm}^{-1}$ increases with the $\mathrm{n}$ value of the $\left[-\mathrm{CH}_{2}-\right]_{n}$. When the UHMWPE characteristics peaks are removed, a broad peak with two shoulders at approximately $1360 \mathrm{~cm}^{-1}$ and $1560 \mathrm{~cm}^{-1}$ can be observed in the Raman spectrum as shown in Fig. 3. These two bands were fitted with two Gaussian functions and can be attributed to the $D$ and $G$ band of hydrogenated amorphous carbon [9]. Although, $\mathrm{sp}^{3} / \mathrm{sp}^{2}$ ratios in the hydrogenated carbon films cannot be derived directly from the Raman spectra, some quantitative information can be extracted from the fitting as the position using full width at half maximum (FWHM) of the $G$ and $D$ peaks as well as the ratio of the $D$ to $G$ peak area - I (D)/I (G). The position of G and D lines, G peak's FWHM and the peak integral intensity ratio I (D)/I (G) are somewhat correlated with the $\mathrm{sp}^{3} / \mathrm{sp}^{2}$ bonding ratio [10].

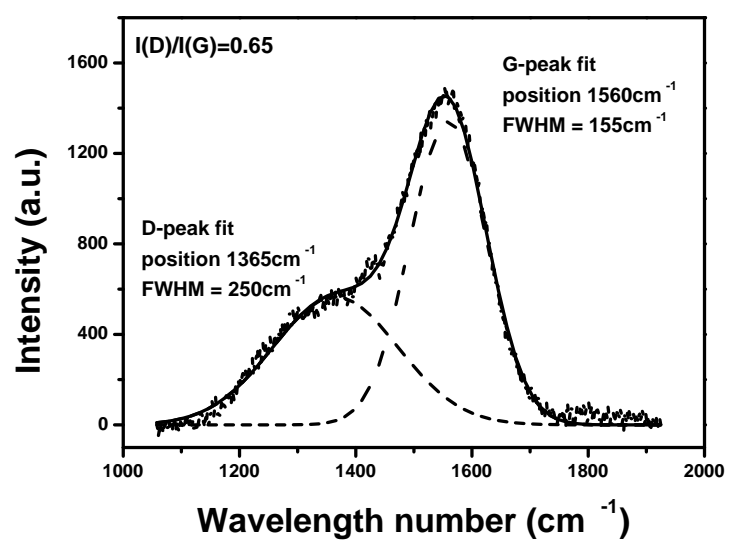

Figure 3. Fitted Raman spectrum after removing the characteristic peaks of UHMWPE.

An important indication of the formation of diamondlike carbon (DLC) layer on the implanted polymer surface can be obtained from the Raman spectrum of implanted UHMWPE which exhibits characteristics typical for the DLC, including a disorder peak (D peak) at $1365 \mathrm{~cm}^{-1}$, and a graphite-like peak (G peak) at $1560 \mathrm{~cm}^{-1}$ with a I (D)/I (G) ratio of about 0.65 .

\subsection{Characterization by XPS}

Currently, the analysis of the X-ray photoelectron spectra (XPS) of the C 1s core level peak has been successfully adopted to estimate the $\mathrm{sp}^{3}$ content in DLC films [11]. The XPS spectra of the UHMWPE obtained in this study was performed using the $\mathrm{Al} \mathrm{K} \alpha$-line, and clearly suggests that the spectral line shape of the DLC C 1s core level is composed of at least two components, with a higher binding shoulder (detected at $285.3 \mathrm{eV}$ ) as can be seen in Fig. 4. Therefore, the fitting of the DLC C $1 \mathrm{~s}$ peaks was performed by using two main components (each being a mixture of a 
Gaussian and a Lorentzian) and by accounting the contribution of the background by the Shirley method. The first component is found at $284.4 \pm 0.1 \mathrm{eV}$ (FWHM $=1.00 \pm 0.05$ $\mathrm{eV}$ ), and corresponds to $\mathrm{sp}^{2}$ carbon atoms, while the second at $285.2 \pm 0.1 \mathrm{eV}(\mathrm{FWHM}=1.10 \pm 0.05 \mathrm{eV})$ corresponds to $\mathrm{sp}^{3}$ carbon atoms. A third peak of much smaller intensity at $286.5 \mathrm{eV}$ has also been added and is attributed to some $\mathrm{C}-\mathrm{O}$ contamination formed at the surface of the samples. This contamination is due to air or residual gas exposure of the dangling bonds, which are formed by polymer chain dehydrogenation [2]. The binding energy values found for the $\mathrm{sp}^{2}$ and $\mathrm{sp}^{3}$ components of the DLC C 1 s spectra are consistent with the binding energies of $284.4 \mathrm{eV}$ and $285.2 \mathrm{eV}$ detected for $\mathrm{C} 1 \mathrm{~s}$ peaks of graphite and diamond respectively. The whole spectrum was deconvoluted into four different contributions at $284.4 \mathrm{eV}, 285.2 \mathrm{eV}, 286.6 \mathrm{eV}$, and $288.3 \mathrm{eV}$. The nitrogen implantation causes an asymmetric broadening of C $1 \mathrm{~s}$ XPS peak towards higher energies. At low nitrogen concentration these peaks could be respectively attributed to $\mathrm{C}-\mathrm{sp}^{2}, \mathrm{C}-\mathrm{sp}^{3}, \mathrm{C}-\mathrm{O}$ and $\mathrm{C}=\mathrm{O}$ or O-C-O chemical bonding formed at the surface [12]. The oxygen present in the chemical structure of the treated polymer is probably due to the oxidation of the long-living radicals, phenomena that is widely reported in the literature. The fitting method for XPS data gives values for $\mathrm{sp}^{2} / \mathrm{sp}^{3}$ ratios within hydrogenated carbon films, which are consistent with the results from other measurements $[11,12]$. In the sequence, the $\mathrm{sp}^{3}$ and $\mathrm{sp}^{2}$ carbon atom content was determined as the ratio of the corresponding peak area over the total $\mathrm{C} 1 \mathrm{~s}$ peak area. Thus, the ratio of $\mathrm{sp}^{3} / \mathrm{sp}^{2}$ hybridized $\mathrm{C}$ in the treated samples was estimated to be about $60 \%$ indicating a formation of hard DLC layer.

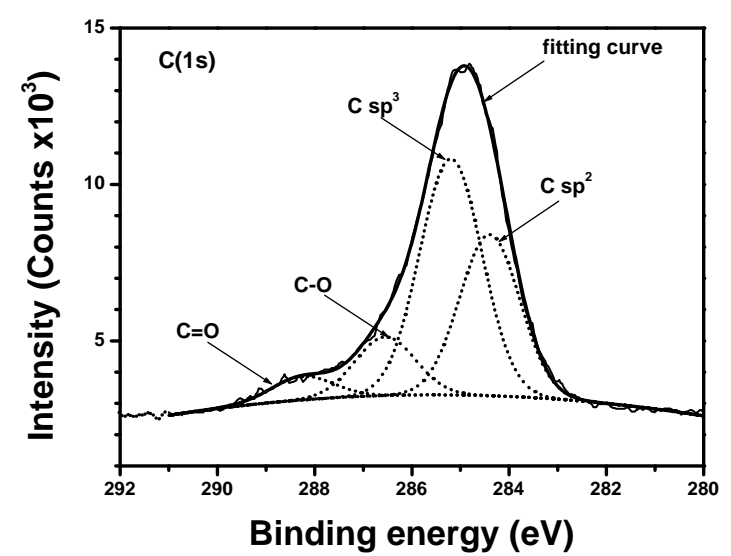

Figure 4. Deconvolution of the XPS C 1s peak of the PIII implanted polyethylene.

The XPS N1s peak for the nitrogen implanted UHMWPE is shown in Fig. 5. A N1s peak (FWHM $=2.9$ $\mathrm{eV}$ ) is found at $399.5 \mathrm{eV}$. Sjostrom et al. [13] have reported the calculations of two model systems in which an $\mathrm{N}$ atom is surrounded by $\mathrm{sp}^{2}$ hybridized carbon atoms or $\mathrm{sp}^{3}$ hybridized carbon atoms. The N1s binding energy for $\mathrm{N}-\mathrm{sp}^{3} \mathrm{C}$ and $\mathrm{N}-\mathrm{sp}^{2} \mathrm{C}$ are $398.3 \mathrm{eV}$ and $400.2 \mathrm{eV}$, respectively. It has also been reported that $\mathrm{N} 1 \mathrm{~s}$ peak position for nitrile $(-\mathrm{C} \equiv \mathrm{N})$ is at $399.4 \mathrm{eV}$ [14]. Therefore, the peak at $399.5 \mathrm{eV}$ in XPS spectrum of the PIII treated polyethylene may be the overlap of the two peaks (398.3 and $400.2 \mathrm{eV}$ ) or corresponds to nitrile. In any way, the implanted nitrogen ions form chemical bonds with the polymer chains instead of forming precipitates by self-clustering (binding energy $\sim 402.0-403.0 \mathrm{eV}$ ) [14].

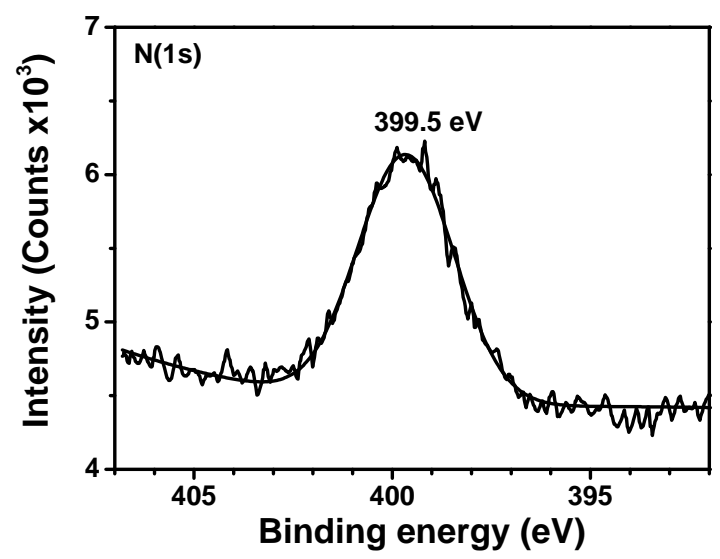

Figure 5. N 1s peak of PIII implanted UHMWPE.

\subsection{Characterization by Nanoindentation te- chnique}

Many polymers are usually too soft for their indentation response to be measured with common nanoindentation testers. Thus, these testers have a natural limited capability for characterizing polymers with regard to hardness and young's module. In this study it was found that roughness level of the unimplanted samples is crucial for hardness characterization of implanted samples. So, well dimensioned grinding and polishing processes are mandatory for a successful execution of nanoindentation analysis. It was found that the refinement must be so good that a mirrored surface of the sample needs to be achieved prior to PIII treatment. Pretreatment 1 was not adequate enough to permit surface hardness characterization, as the light reflexion of the unimplanted surface was minimal. However, pretreatment 2 has shown to be adequate in providing samples with mirrored surface in such a way that hardness and elastic modulus could be measured within acceptable depths. For sequences details, see table 1 . The load values are very important in nanoidentation technique, especially when we are dealing with soft material as polymers. In this experiment the load was chose as light as 4 grams in order to avoid less effect from the bulk of the substrate. The penetration depth was from $300 \mathrm{~nm}$ to $2200 \mathrm{~nm}$. The bulk effect would be significant for a high penetration depth, reducing the observed hardness value [15]. 
Even with $4 \mathrm{~g}$ load the effect of the substrate bulk could not be avoided. So the conclusion is that lighter loads should be used until the limit where hardness measurement were independent of the substrate (type and roughness) and, in this way, uniquely attributed to DLC formed on the polymer surface. But we shall have in mind that there is a critical balance between the load value and measurement error in such a way that smaller the load higher the measurement error.

Another point that needs to be mentioned is the necessity of performing multiple measurements for each depth. The measurement error inherent to nanoidentation technique is high and, in this way, statistical data treatment based on mean values is necessary for results with a good level of confidence.

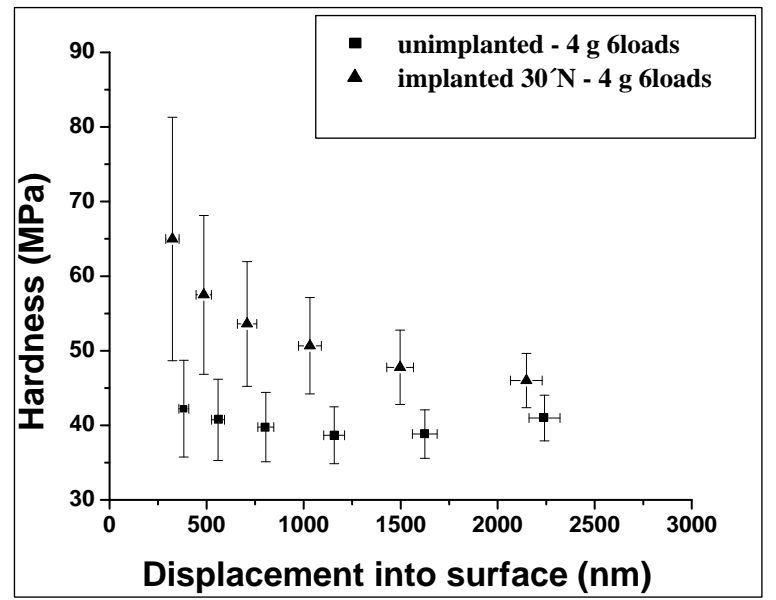

Figure 6. Hardness of uninplanted and PIII implanted UHMWPE.

As illustrated in Fig. 6, we can see that PIII treatment has a pronounced hardening effect on UHMWPE. In fact the hardening level depends on the depth of the measurement and closer the surface we measured it more pronounced was the hardening effect. This result was already predictable because improvement on polymer mechanical properties induced by ion implantation is largely due to the formation of a three-dimensional covalent bonds network among the polymer chains. As the ions are implanted from the surface to the interior of the material, intensity of cross-linking bonds are expected to decrease in the same direction. This conclusion is supported by the literature [16].

In this study, it was found that within depths of the order of $0.5 \mu \mathrm{m}$ the hardness was improved by $50 \%$. The hardness improvement is higher at distances closer to the surface but it was very difficult to measure it cause of the polymer characteristics already discussed. Therefore, significant surface modifications are confined to a very thin layer $(<1 \mu \mathrm{m})$ beneath the surface. Fig. 7 presents the enhanced elastic modulus for modified UHMWPE, which also shows a tendency to get higher at the very surface of the sample.

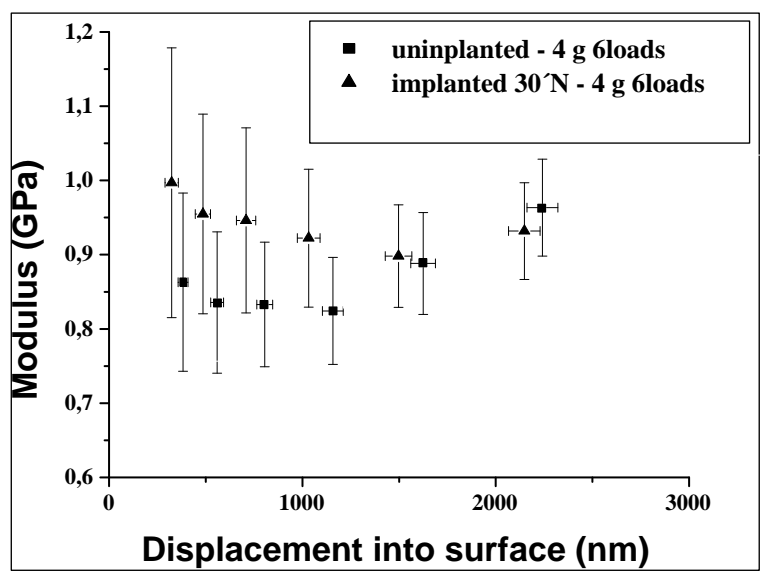

Figure 7. Elastic modulus of uninplanted and PIII implanted UHMWPE.

\section{Conclusions}

Plasma immersion ion implantation has been demonstrated to be an effective treatment for surface modification of UHMWPE. The nitrogen ion bombardment has caused a significant decreasing of the hydrogen content in the polymer surface. That phenomenon represents, in fact, a structure modification of the polymer chemical chains. The structure modification consists of bombardment of the polymer surface by nitrogen energetic ions, in such a way that the ions cause bond breakage or molecular chain scission by displacing atoms from polymer chains. The subsequent reactions lead to free radical formation and new and stronger cross-linked bonds. So, new chemical species are created on polymer surface, which leads to better tribological properties. The XPS results show that the initial surface layer has in fact been converted into hydrogenated amorphous carbon a new layer obtained on the polymer surface that is DLC.

Raman spectroscopy of treated samples has shown an enhanced luminescence background and a G and D bands centered at $1560 \mathrm{~cm}^{-1}$ and $1365 \mathrm{~cm}^{-1}$, respectively.

XPS C 1s core level spectra of the modified layer were used for evaluation of the content of $\mathrm{sp}^{3}$ and $\mathrm{sp}^{2}$ carbon atoms at the polymer surface. The estimated ratio of $\mathrm{sp}^{3} / \mathrm{sp}^{2}$ hybridized $\mathrm{C}$ in the treated samples strongly indicated the formation of DLC layer.

It has been proven that PIII can also significantly improve the surface hardness and elastic modulus of UHMWPE and one of the important conclusions of this work is that the improvement of the grounding and polishing processes permits easier evaluation of these mechanical properties at the surface of the material.

The induced cross-linking can effectively retard molecular structure reconstruction or alignment of lamellar crystallites in UHMWPE, thus giving rise to high resistance to initiation and propagation of micro-crack. With the crosslinked bonds, the molecular chain mobility is reduced and the polymer surface presents therefore a higher hardness and 
an improved inertness. For UHMWPE prostheses application, the cross-linked bonds are usually obtained by gamma radiation sterilization process. But sterilization in air may be particularly harmful, because it may initiate a long-term oxidative process, which has a negative impact on the implant's mechanical properties. Nitrogen PIII process eliminates these disadvantages while keeping the benefits of cross-linked bonds.

\section{Acknowledegments}

One of the authors, A. R. Marcondes, wishes to thank Capes for his fellowship. FAPESP has also financially supported this work. Another author, G. F. Gomes, gratefully acknowledges a post-doctoral fellowship from FAPESP.

\section{References}

[1] D. Lwise, D. J. Trantolo, D. E. Altobelli, M. J. Yaszemski, J. D. Gresses, and E. R. Schwarts, Encyclopedic Handbook of Biomaterials and Bioengineering A, 2, 1759 (1995).

[2] J. S. Chen, S. P. Lau, Z. Sun, B. K. Tay, G. Q. Yu, F. Y. Zhu, D. Z. Zhu, and H. J. Xu, Surf. Coat. Techol. 138, 33 (2001).

[3] W. Shi, X. Y. Li, and H. Dong, Wear, 250, 544 (2001).

[4] D. Ikeda, M. Ogawa, Y. Hara, Y. Nishimura, O. Odusanya, K. Azuma, S. Matsuda, M. Yatsuzuka, and A. Murakami, Surf. Coat. Technol. 156, 301 (2002).
[5] M. Konuma, Film Deposition by Plasma Techniques, Springer, Berlin, p. 171 (1992).

[6] M. Ueda, L. A. Berni, J. O. Rossi, J. J. Barroso, A. F. Beloto, and E. Abramof, Surf. Coat. Technol. 136, 28 (2001).

[7] A. Valenza, A. M. Visco, L. Torrisi, and N. Campo, Polymer, 45, 1707 (2004).

[8] D. Lin-Vien, N. B. Colthup, W. G. Fateley, and J. G. Grasselli, Infrared and Raman Characteristic Frequencies of Organic Molecules, Academic Press, Inc (1991).

[9] J. Y. Chen, L. P. Wang, K. Y. Fu, N. Huang, Y. Leng, Y. X. Leng, P. Yang, J. Wang, G. Wan, H. Sun, X. Tian, and P. K. Chu, Surf. Coat. Technol. 156, 289 (2002).

[10] J. Robertson, Mat. Sci. Eng. Reports 37, 129 (2002).

[11] P. Merel, M. Tabbal, M. Chaker, S. Moisa, and J. Margot, Appl. Surf. Sci. 136, 105 (1998).

[12] J. Filik, P. W. May, S. R. J. Pearce, R. K. Wild, and K. R. Hallam, Diamond Rel. Mat. 12, 974 (2003).

[13] H. Sjostrom, S. Stafstrom, M. Boman, and J. E. Sundgen, Phys. Rev. Lett. 75, 1331 (1995).

[14] A. Toth, T. Bell, I. Bertoti, M. Mohai, and B. Zelei, Nucl. Instr. and Meth. B 148, 1131 (1995).

[15] H. C. Ong, R. P. H. Chang, N. Baker, and W. C. Oliver. Surf. Coat. Technol. 89, 38 (1997).

[16] H. Dong and T. Bell, Surf. Coat. Technol. 111, 29 (1999). 